\title{
The impact of winter feed type on intestinal microbiota and parasites in honey bees
}

\author{
Paul D’Alvise ${ }^{1}$, Franziska BöHme ${ }^{2}$, Marius Cosmin CodreA $^{3}$, Alexander SeItz ${ }^{4}$, \\ Sven NAHNSEN ${ }^{3}$, Mieke Binzer ${ }^{1}$, Peter Rosenkranz ${ }^{2}$, Martin Hasselmann ${ }^{1}$ \\ ${ }^{1}$ Institute for Animal Science, University of Hohenheim, Garbenstraße 17, 70599, Stuttgart, Germany \\ ${ }^{2}$ Apicultural State Institute, University of Hohenheim, August-von-Hartmann-Str. 13, 70599, Stuttgart, Germany \\ ${ }^{3}$ Quantitative Biology Center, University of Tübingen, Auf der Morgenstelle, 72076, Tübingen, Germany \\ ${ }^{4}$ Center for Bioinformatics, University of Tübingen, Sand 14, 72076, Tübingen, Germany
}

Received 12 July 2017 - Revised 4 September 2017 - Accepted 27 September 2017

\begin{abstract}
The intestinal microbiota of honey bees consists of only few bacterial species and may have effects on health and pathogen resilience. Honey is usually harvested and replaced by sugar syrup. We hypothesized that replacing honey may change the composition of the intestinal microbiota, and therefore compromise pathogen resilience. Fifteen colonies were fed with wheat starch syrup, sucrose syrup, or blossom honey. 16S-based bacterial community analysis was performed on three individuals per hive in summer and winter, and Nosema ceranae and Crithidia/Lotmaria levels were assessed by qPCR. Seasonal differences in the intestinal microbiota and $N$. ceranae were found; however, microbiota and parasite levels were very similar between the feed types. Rhizobiales and Bifidobacteria were found to be increased in the bees that had received honey or wheat starch syrup, as compared to sucrose syrup. In conclusion, intestinal microbiota and parasites were found to be largely unaffected by the winter feed type.
\end{abstract}

\section{honey / syrup / microbiota / Nosema / Crithidia}

\section{INTRODUCTION}

During the past decades, the global population of honey bees has been suffering from an increased rate of colony losses during winter and a multifactorial syndrome termed colony collapse disorder (Genersch et al. 2010; vanEngelsdorp et al. 2009). Winter losses are often connected with high infestation with the parasitic mite Varroa destructor, which acts as a vector for viruses, in combination with intrinsic and envi-

Electronic supplementary material The online version of this article (https://doi.org/10.1007/s13592-017-0551-1) contains supplementary material, which is available to authorized users.

Corresponding author: P. D'Alvise, paul.dalvise@uni-hohenheim.de Manuscript editor: Stan Schneider ronmental factors that affect honey bee health (Di Prisco et al. 2016; Wilfert et al. 2016), while the causes of colony collapse disorder remain elusive. With the emerging paradigm that immune response and pathogen resilience of an (insect) host is influenced by its intestinal microbiota (Ryu et al. 2008; Silverman and Paquette 2008), it may be worthwhile to address if, and to what extent, health and disease resistance of the honey bee is influenced by its intestinal microbiota, and whether the microbiota is influenced by beekeeping practices.

Honey bees have a remarkably simple intestinal microbiota, which consist of only few anaerobic or microaerophilic core species (Engel et al. 2013; Martinson et al. 2011; Moran 2015). Some of these species, Gilliamella apicola, Snodgrassella alvi, Parasaccharibacter apium, some Lactobacillus spp., and a Bifidobacterium species are 
specific to corbiculate bees and appear to have coevolved with their hosts (Corby-Harris 2016; Koch et al. 2013; Kwong et al. 2017; Vásquez et al. 2012). Frischella perrara and Bartonella apis are only found in the Apis genus. The typical microbiota is acquired by newly emerged bees by oral transmission through trophallaxis (mostly lactic acid bacteria) and by fecal-oral transmission through contact with other workers or hive material (Martinson et al. 2012; Powell et al. 2014). Recently, the normal honey bee microbiota was shown to establish a lowered $\mathrm{pH}$ level and an oxygen gradient within the gut and provide its host with short-chain fatty acids, resulting in higher host weight gain, as compared to an atypical lab-acquired microbiota (Zheng et al. 2017).

Genomic data indicates that the numerically dominant species $G$. apicola is capable of degrading and processing diverse carbohydrates, such as pectin, that occur in pollen cell walls, while it seems to lack key enzymes in the TCA cycle, which may force it to ferment carbohydrates into carboxylates (Kwong et al. 2014; Moran 2015). Besides, G. apicola is able to degrade monosaccharides that would otherwise be toxic for the bees (Zheng et al. 2016). In contrast, the second most abundant species $S$. alvi may be unable to process complex carbohydrates or sugars and may therefore only utilize carboxylates via its functional TCA cycle. Thus, the metabolic capabilities of the two dominant species seem to be complementary, likely as a result of a long history of coevolution. It has to be mentioned that, while the honey bee intestinal microbiota comprises only few species, there is a high degree of diversity within these species, and different strains of one species can have different metabolic capabilities (Engel et al. 2014; Kwong et al. 2014).

A disease-reducing effect of honey bee gut bacteria was demonstrated in larvae, where beeassociated lactic acid bacteria were shown to inhibit Paenibacillus larvae, the causative agent of American foulbrood, and in vitro experiments indicated antagonistic effects against transiently occurring microbes (Forsgren et al. 2010; Vásquez et al. 2012). P. apium was demonstrated to increase resistance to Nosema infection (Corby-Harris 2016), and lower N. ceranae levels after experimental infection were found in honey bees fed with Lactobacillus and Bifidobacterium strains (Baffoni et al. 2016). In bumblebees, infection with intestinal parasites (Crithidia sp.) was found to be negatively correlated with presence of symbiotic bacteria (Gilliamella sp.) (Cariveau et al. 2014; Koch and Schmid-Hempel 2011). Similar findings of bacterial insect symbionts antagonizing pathogenic bacteria were reported from, e.g., locusts and aphids (Dillon et al. 2005; Scarborough et al. 2005). On the other hand, there is evidence that not all ubiquitous bacterial species of the honey bee intestine are purely beneficial. F. perrara was shown to cause tissue necrosis in the pylorus, its principal attachment site, probably by producing a colibactin-like substance (Engel et al. 2015a; Engel et al. 2015b). An increase of $F$. perrara and simultaneous decrease of $S$. alvi, which was caused by an aged natural diet, was shown to be associated with higher establishment of Nosema sp. and increased mortality (Maes et al. 2016). It is not known whether F. perrara is merely a pathogen, or whether it also has beneficial properties for its host. Also, the other bacterial symbionts' roles, adverse or beneficial, are far from being elucidated, and the simple concept of a bacterial species being basically either beneficial or deleterious does not always apply; $S$. alvi, for example, was shown to promote establishment of Lotmaria passim when administered together with the parasite (Schwarz et al. 2016). However, since honey bees are easy to work with and their intestinal microbiota is consistently composed of the same few species, it is well suited to become a model system for understanding host-microbiota interactions.

During their evolution towards a eusocial lifestyle, honey bees seem to have shifted tasks of the originally individual immune responses to the community level. In return for fewer immunerelated genes, as compared to non-social insects, and reduced lysozyme production, honey bees deploy propolis, an antimicrobial mixture of plantderived saps and resins, provide their larvae with royal jelly, which is rich in antimicrobial peptides, and produce honey as winter stock, which has antibacterial, antifungal, and antiviral properties (Anderson et al. 2011; Evans et al. 2006; Kunieda et al. 2006; Romanelli et al. 2011; Viuda-Martos et al. 2008). In managed colonies, honey is usually 
harvested and replaced with sucrose or corn starch-based syrup. Unlike these replacement diets, honey does not only consist of sugars, but also contains small fractions of enzymes and secondary metabolites that mediate the aforementioned antipathogen effects (da Silva et al. 2016). Feeding with replacement diets, as compared to honey, results in gene expression differences, indicating that the replacement diets may have a different nutritive value (Wheeler and Robinson 2014). However, it is not known whether and how this may affect the intestinal microbiota and phenotypic traits such as longevity, immunocompetence or pathogen tolerance. An older study comparing two replacement diets with honey did not find any significant effects on post-winter cluster size, brood rearing or honey production in the following year (Severson and Erickson 1984).

The aim of the present study was to reveal whether the composition of the honey bee intestinal microbiota is changed by replacement winter feed types, as compared to honey, the natural winter stock. For this purpose, honey bees from 16 hives were sampled before and after receiving and consuming honey and two different syrup types, and the bacterial communities of 93 single bees were profiled. In addition, we quantified two common eukaryotic intestinal parasites by quantitative PCR, the microsporidian Nosema ceranae and the two very closely related trypanosomatids Crithidia mellificae and Lotmaria passim, to elucidate if changes in the microbiota may be associated with parasite abundance. Among the many honey bee parasites, these two species were selected, since they are often present in healthy bees, live in close contact with the intestinal bacterial community, and may be influenced by presence of certain bacterial species or strains (Baffoni et al. 2016; Cariveau et al. 2014; Corby-Harris 2016; Koch and Schmid-Hempel 2011).

\section{MATERIAL AND METHODS}

Experimental hives, treatments, and sampling Sixteen honey bee colonies in polystyrene Miniplus hives were set up in June by making artificial swarms from five donor colonies and distributing the bees to the treatment groups in a randomized block design. All colonies filled three boxes and were kept in the same apiary. The colonies were treated against mites using one strip of Bayvarol (Bayer AG, Leverkusen, Germany) per hive for 6 weeks from mid-August until end of September. In late August, forager bees, identified by corbicular pollen, were sampled from all hives. Two days after the first sampling, the colonies were fed with ten liters (4 consecutive portions of 2.5 1) of wheat starch syrup (Graze, Weinstadt, Germany), sucrose-derived syrup (Api Invert, Südzucker, Germany), or locally produced spring blossom honey. In early December, all hives were treated against mites with $30 \mathrm{~mL}$ of $3.5 \%(\mathrm{~m} / \mathrm{v})$ oxalic acid. One hive was found dead in December, while all other colonies survived until spring and had winter feed left over. Post-winter samples of winter bees were retrieved from inside the hives on a sunny day in February, before spring bees emerged and before the first forage was available.

Sample storage and DNA extraction Bee samples were immediately chilled on ice and transported to the laboratory within $2 \mathrm{~h}$, where they were stored at $-80^{\circ} \mathrm{C}$ until DNA extraction. Single, complete gastrointestinal tracts including honey bladders were removed from thawed bees by pulling out the terminal abdominal segment with forceps and were DNA-extracted using the Machery-Nagel NucleoSpin Soil kit according to the manufacturer's protocol. Cell lysis was performed on a FastPrep 24 (MP Biomedicals, Illkirch, France) at $5.5 \mathrm{~m} / \mathrm{s}$ for $60 \mathrm{~s}$, and DNA was eluted twice in $50 \mu \mathrm{L}$ elution buffer, resulting in DNA concentrations ranging from 30 to $60 \mathrm{ng} / \mu \mathrm{L}$. Quality and quantity of the extracts were assessed using an Optizen NanoQ photometer (Mecasys, Daejeon, Korea) and a Qbit fluorimeter (Thermo Fisher Scientific, Waltham MA, USA). For every colony and sampling point, three extracts of single bee gastrointestinal tracts, a total of 93 samples, were submitted to $16 \mathrm{~S}$-gene amplicon sequencing analysis.

\subsection{S-gene amplicon sequencing and analysis}

16S-gene amplicons were generated using 25 ng template DNA in a $20-\mu \mathrm{L}$ PCR reaction targeting the V3-V4 region of the $16 \mathrm{~S}$ rRNA 
gene. Sequencing libraries were generated with the Nextera XT DNA library preparation kit (Illumina, San Diego, USA). Amplicon sequencing was performed on an Illumina MiSeq v2 system and generated 26,005,981 paired-end reads of $2 \times 250 \mathrm{bp}$. The procedure was based on the protocol suggested by Illumina. The data were de-multiplexed using CASAVA (version 1.8.2, Illumina, Inc., 2010). The adapters were trimmed with Skewer (version 0.1.116 (Jiang et al. 2014)), and quality control was performed using FastQC (version 0.10.1, Babraham Bioinformatics). Of the reads, $78.39 \%$ had Phred quality scores over 30; thus, 20.2 million reads were normalized to 217,500 reads per sample and used in the analysis. Alignment, taxonomic binning, and analysis of the reads were carried out using MALT (version 0.3.8) and MEGAN (version 6.7.0) (Herbig et al. 2016; Huson and Weber 2013). The taxonomic binning in MALT and MEGAN is based on the naive least common ancestor (LCA) algorithm. Thus, a read is placed on the lowest node of the NCBI taxonomy that lies above all taxa with a significant alignment. For the comparison, the counts were normalized to the smallest number of reads of any of the samples. All generated sequence datasets were submitted to the NCBI Sequence Read Archive: BioProjectID PRJNA382070, accessions SAMN06699129SAMN06699221.

\subsection{Quantification of Nosema ceranae and the trypanosomatids by qPCR}

The Nosema ceranae infection in the sampled bees was assessed by quantitative PCR (qPCR), using the same DNA extracts as for the 16S-gene amplicon sequencing. Template DNA $(0.8 \mu \mathrm{L}$, diluted $1: 2)$ was used in $10-\mu \mathrm{L}$ reactions with the primers NCF203 (5'-CAGTTATGGGAAGT AATATTATATTG) and NCR253 (5'-TTGA TTTGCCCTCCAATTAATCAC) (Cox-Foster et al. 2007), using the Platinum SYBR Green qPCR SuperMix-UDG (Invitrogen, Carlsbad CA, USA). Thermal amplification (thermal profile: 2-min initial denaturation at $95^{\circ} \mathrm{C}, 40$ cycles of $15 \mathrm{~s}$ at $95{ }^{\circ} \mathrm{C}, 15 \mathrm{~s}$ at $52{ }^{\circ} \mathrm{C}, 10 \mathrm{~s}$ at $64{ }^{\circ} \mathrm{C}$ (fluorescence read), $15 \mathrm{~s}$ at $72{ }^{\circ} \mathrm{C}$ ) was carried out on a BioRad CFX96 C100 thermocycler. Specific melting temperature of the amplicons was $68^{\circ} \mathrm{C}$. For quantification of Crithidia/Lotmaria, the primers SEF (5'-CTTTTGGTCGGTGG AGTGAT) and SER (5'-GGACGTAATCGGCA CAGTTT) were used (Meeus et al. 2010) (thermal amplification profile: 2-min initial denaturation at $95{ }^{\circ} \mathrm{C}, 40$ cycles of $15 \mathrm{~s}$ at $95{ }^{\circ} \mathrm{C}, 30 \mathrm{~s}$ at $58^{\circ} \mathrm{C}$ (fluorescence read)). Specific melting temperature of the amplicon was $83{ }^{\circ} \mathrm{C}$. Specificity of both assays was confirmed by Sanger sequencing of the amplicons. Purified amplicons of both assays were quantified by microdroplet spectrometry and fluorimetry as described above, serially diluted $1: 10$, and used as standards for qPCR. The number of amplicon molecules per microliter of standard was calculated from the measured DNA concentration and their molecular weight (DNA molecular weight calculator www.bioinformatics. com/sms2/dns_mw.html). The detection limit was 126 template molecules $/ \mu \mathrm{L}$, and the linear dynamic range extended to $1.9 \times 10^{9} / \mu \mathrm{L}(\mathrm{Cq}=5)$ for the $N$. ceranae assay. The detection limit for the trypanosomatids was $62 / \mu \mathrm{L}$ and the linear dynamic range extended to $1.5 \times 10^{9} / \mu \mathrm{L}(\mathrm{Cq}=2.5$ ). The PCR efficiency was $93.7 \%$ ( $N$. ceranae) and $85.6 \%$ (Crithidia/Lotmaria), and the regression coefficients were 0.9955 and 0.9921 , respectively.

\subsection{Statistics}

All statistics were calculated using JMP version 12.0.2 (SAS, Cary, NC, USA). For the 16S-based bacterial community analyses, read numbers specific for a bacterial taxon were divided by the total number of reads from the respective sample to obtain the relative abundance of the respective taxon. The residuals of bacterial relative abundances were found to be not normally distributed (Shapiro-Wilk test); thus, the overall differences between groups were analyzed by a Wilcoxon/Kruskal-Wallis test, and pairwise comparisons of treatment groups were done with a paired Wilcoxon tests. $P$ values and chi-square approximations given in the text were derived from the Wilcoxon/ Kruskal-Wallis tests. Nonparametric correlations of bacterial group abundances were examined, based on read numbers, by calculating 


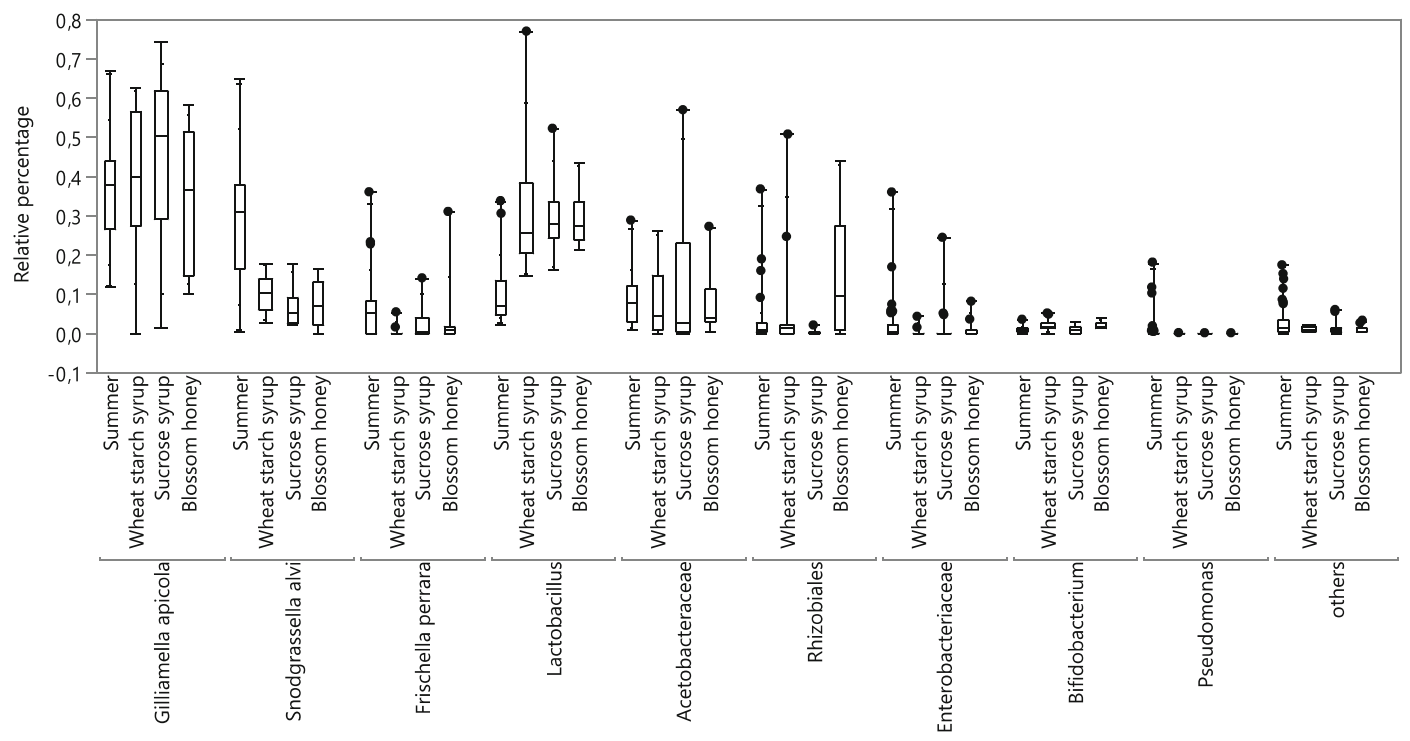

Figure 1. Quantile plot of the relative abundances of the major bacterial groups in the summer samples and after overwintering on syrup or honey. The boxes indicate 25th, 50th (median), and 75th percentiles, and whiskers indicate the data range. Dots indicate data points identified as outliers $(25$ th or 75 th percentiles $+1.5 \times$ interquantile range). If significant differences were found between the abundances of a bacterial group, capital letters denote groups of significance and asterisks denote significance levels $(* P<0.05, * * P \leq 0.01, * * * P \leq 0.001)$.

Spearman's rank correlation coefficient (Spearman's rho). The significance of clustering within the principal coordinate analyses was tested using the triangulation test provided in MEGAN (Huson and Weber 2013). Differences between parasite levels, as detected by
qPCR, were tested by a chi-square $\left(\chi^{2}\right)$ test based on the numbers of individual bees that were positive or negative for the respective parasite and $P$ values were derived from the chi-square value. In all statistical analyses, significance was stated if $P$ was below 0.05 .

Table I. Extreme values and medians of the relative abundances of the major bacterial groups

\begin{tabular}{|c|c|c|c|c|c|c|}
\hline & \multicolumn{3}{|l|}{ Summer } & \multicolumn{3}{|l|}{ Winter } \\
\hline & Minimum & Maximum & Median & Minimum & Maximum & Median \\
\hline Gilliamella apicola & $11.8 \%$ & $67.0 \%$ & $37.8 \%$ & $0.16 \%$ & $74.3 \%$ & $38.5 \%$ \\
\hline Snodgrassella alvi & $0.60 \%$ & $64.7 \%$ & $30.8 \%$ & $0.11 \%$ & $18.0 \%$ & $7.5 \%$ \\
\hline Frischella perrara & $0.01 \%$ & $35.9 \%$ & $5.1 \%$ & $0.009 \%$ & $30.9 \%$ & $0.1 \%$ \\
\hline Lactobacillus spp. & $2.32 \%$ & $33.7 \%$ & $7.1 \%$ & $14.6 \%$ & $76.9 \%$ & $27.0 \%$ \\
\hline Acetobacteraceae spp. & $0.76 \%$ & $28.7 \%$ & $7.9 \%$ & $0.028 \%$ & $56.9 \%$ & $3.7 \%$ \\
\hline Rhizobiales spp. & $0.02 \%$ & $36.7 \%$ & $0.77 \%$ & $0.019 \%$ & $50.7 \%$ & $1.1 \%$ \\
\hline Enterobacteriaceae spp. & $0.02 \%$ & $35.9 \%$ & $0.26 \%$ & $0.005 \%$ & $24.3 \%$ & $0.03 \%$ \\
\hline Bifidobacterium spp. & $0.14 \%$ & $3.4 \%$ & $0.74 \%$ & $0.008 \%$ & $5.0 \%$ & $1.7 \%$ \\
\hline Pseudomonas spp. & $0.002 \%$ & $18.0 \%$ & $0.01 \%$ & $0 \%$ & $0.19 \%$ & $0.002 \%$ \\
\hline Other bacteria & $0.15 \%$ & $17.3 \%$ & $1.1 \%$ & $0.19 \%$ & $5.9 \%$ & $0.70 \%$ \\
\hline
\end{tabular}




\section{RESULTS}

Bacterial community analysis by $16 \mathrm{~S}$-gene amplicon sequencing revealed that the intestinal microbiota of the sampled bees was mainly composed of G. apicola, S. alvi, Lactobacillus spp., F. perrara, Acetobacteraceae (Saccharibacter, Parasaccharibacter, Commensalibacter), Rhizobiales (Bartonella), Enterobacteriaceae, and Bifidobacterium spp. (Figures 1 and S1). Although all of these bacterial groups were represented in any of the 93 individuals sampled, there was a large inter-individual variation in the size of the fractions. Even for the most dominant species, $G$. apicola, the relative abundance ranged between 0.16 and $74 \%$ (Table I). When the $G$. apicola fraction was low, lactobacilli and Acetobacteraceae were most abundant. Correspondingly, there was a significant negative correlation between $G$. apicola and Acetobacteraceae $(P<0.001)$, and also the pathobiont $F$. perrara was negatively correlated with its close relative G. apicola $(P=0.0032$, Table II). Generally, there were fewer $S$. alvi than $G$. apicola found in the samples; however, in some individuals, one of the species was found abundantly although their assumed symbiotic partner was practically absent (Figure 2). The abundance of Lactobacillus spp. was negatively correlated with most of the other bacterial groups, except the bifidobacteria, which showed a significant positive correlation $(P<0.001)$. In contrast, the Rhizobiales group (Bartonella) showed no correlation with any of the other groups except a positive correlation with the bifidobacteria $(P=0.0015)$. Finally, Enterobacteriaceae, Pseudomonas spp., and other bacteria showed positive correlations with each other ( $P<0.001, P=0.0031, P<0.001$, respectively).

The most striking differences in intestinal bacterial communities were found between summer and winter samples (Figure 3). In large terms, S. alvi was more abundant in the summer samples (derived $\chi^{2}=42.4 ; P<0.001$ ), while Lactobacillus spp. were more abundant in winter (derived $\left.\chi^{2}=56.3 ; P<0.001\right)$. F . perrara fractions were generally larger in the summer forager bees $(P<0.001)$. Ten of the sampled summer bees had F. perrara fractions over $10 \%$, while this was 


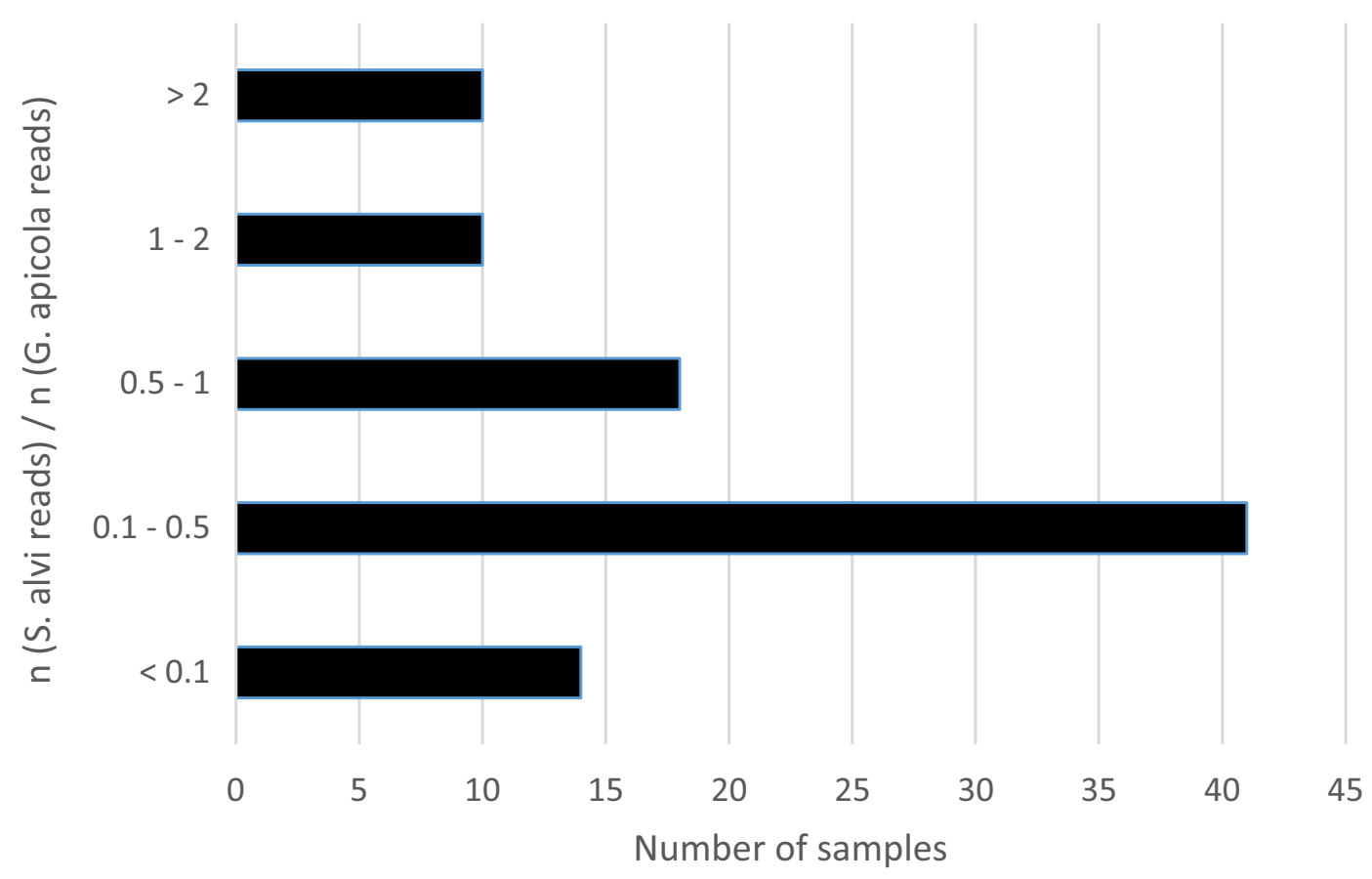

Figure 2. Ratios of Snodgrassella alvi/Gillimella apicola reads calculated separately for single bee samples. The median $S$. alvi/G. apicola ratio is 0.34 .

only two bees in winter. Similarly, F. perrara fractions below $1 \%$ were found in 16 (summer) and 30 bees (winter). Pseudomonas sp. was abundant during summer (up to $18 \%$ ) in some individuals, while it was much less abundant in the winter samples $(\leq 0.016 \%)$. The fraction of bacteria other than the aforementioned groups, which contains all transient species, was greater in summer foragers (derived $\chi^{2}=8.5 ; P=0.0035$ ). Also, the variability of the gut bacterial communities was different between the seasons (Figure 1); the size of $G$. apicola fractions was more consistent in the summer samples, while the size of the lactobacilli fractions was more uniform in winter.

There was a pronounced seasonal difference in $N$. ceranae levels; in summer, $N$. ceranae was detected in all bees, while in winter, $N$. ceranae was only detected in 9 of 45 bees sampled (Table III, $\left.\chi^{2}=79.1 ; P<0.001\right)$. The trypanosomatids (C. mellificae/L. passim) were detected in 39 of the 48 summer bees and in 24 of the 45 winter bees (Table IV, $\chi^{2}=8.4 ; P=0.0037$ ). Medium and high levels of trypanosomatids were found only in five of the summer foragers. The bees sampled from the colony that died during early winter showed no obvious differences in their microbial communities or parasite levels, as compared to the rest of the summer samples.

As for the effects of the different carbohydrate replacement diets on the intestinal microbiota, the composition of the bacterial communities in the late winter samples was, in large terms, not significantly different between the experimental groups (Figures 1 and 4), indicating that the two replacement diets did not cause major changes in the microbiota, as compared to honey or to each other. However, the fractions of Rhizobiales (Bartonella) and Bifidobacteria were reduced in the colonies that received sucrose syrup as winter feed (derived $\chi^{2}=13.9$ and $8.4 ; P=0.001$ and 0.0147$)$. The Rhizobiales fraction (mean values) was highest in the colonies fed with honey $(14.6 \%)$ and $5.9 \%$ in the colonies fed with wheat starch syrup, while it was reduced to $0.44 \%$ in the colonies that had received sucrose syrup. The Bifidobacteria fraction was reduced to $0.97 \%$ after overwintering on sucrose syrup, as compared 


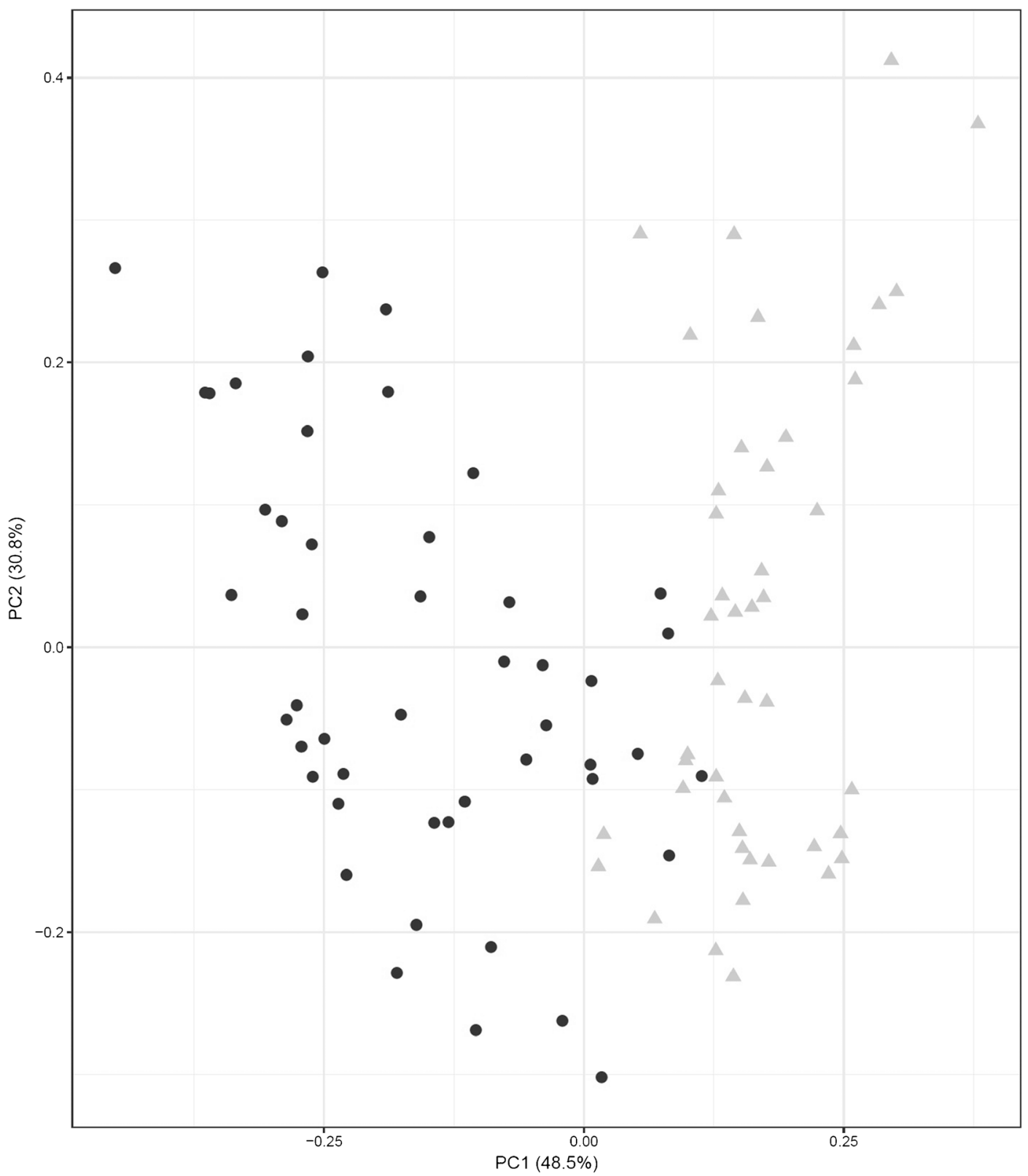

Figure 3. Principal coordinate analysis of the bacterial communities on genus level in all samples using the BrayCurtis method as distance metric. Triangles represent the samples collected in winter, and circles represent the samples collected in summer. A triangulation test indicated that the separation of clusters was significant $(P<0.05)$.

to $1.9 \%$ (honey) and $2.2 \%$ (wheat starch syrup). The different winter feed types were not associated with any significant differences in $N$. ceranae or trypanosomatid infection ( $N$. ceranae: $\chi^{2}=0.85 ; P=0.6549 ;$ C. mellificae/L. passim: $\left.\chi^{2}=1.61 ; P=0.4466\right)$.

\section{DISCUSSION}

The intestinal microbiota found in this study was composed of the same core species reported previously as typical constituents of the honey bee microbiota (Engel et al. 2013; Kwong and Moran 
Table III. Levels of $N$. ceranae in the sampled bees $\left(\log _{10}(\mathrm{n} / \mu \mathrm{L})\right.$ for the summer and winter samples

\begin{tabular}{lllllll}
\hline Nosema level $\log 10(\mathrm{n} / \mu \mathrm{L})$ & Not detected $<2.2$ & Low 2.2-5 & Medium 5-7 & High 7-9 & Very high $>9$ & Total \\
\hline Summer foragers & 0 & 16 & 9 & 20 & 3 & 48 \\
Winter bees overall & 36 & 3 & 2 & 2 & 2 & 45 \\
Blossom honey & 11 & 3 & 0 & 1 & 0 & 15 \\
Wheat starch syrup & 13 & 0 & 0 & 1 & 1 & 15 \\
Sucrose syrup & 12 & 0 & 2 & 0 & 1 & 15 \\
\hline
\end{tabular}

The winter samples are further distinguished by treatment (feed type)

2016). It is striking that this specific bacterial community is conserved between honey bee populations on different continents and in different climates. The low number of species and their ubiquitous occurrence emphasizes the honey bee as a simple model system for mechanistic studies on microbiota and bacteria-host interactions. Such studies are needed to understand the functional role of the single microbial species within the community and within their host, and to elucidate why this particular microbial ecosystem remains so stable. Beyond honey bee physiology, the simple, surveyable bacterial community of the honey bee guts would allow inexpensive in vivo studies on microbial ecology that could generate novel insights into more complex bacterial communities.

The fact that the microbiota of the honey bee gut contains so few species, as compared to other animals, suggests an efficient mechanism that prevents invasion by other bacterial species. Kwong et al. (2014) found genes for components of a type VI secretion system in the genome of $S$. alvi, which may be involved in bacterial antagonism though cell-to-cell contact. Since the intestinal mucosa is coated by a biofilm of $S$. alvi, this bacterium could be able to efficiently antagonize invading bacteria throughout the community (Schluter and Foster 2012). We found that Lactobacillus spp. were negatively correlated with $S$. alvi and with most of the other bacterial groups. This may be indicative of an underexplored antagonistic potential of the bee-specific lactobacilli, as indicated by previous studies (Forsgren et al. 2010; Vásquez et al. 2012). However, the occurrence of lactobacilli and bifidobacteria was clearly positively correlated, which may reflect their similar substrate requirements or, possibly, a mutualistic relation. The positive correlations found between the non-specific members of the microbial community, i.e., Enterobacteriaceae, Pseudomonas, and other bacteria, along with their pronounced negative correlations with the lactobacilli might indicate that these species can thrive when the specific bacterial community is perturbed and fewer Lactobacillus spp. are present.

The differences in bacterial community composition found between summer foragers and winter bees, namely less $S$. alvi and more Lactobacillus spp. in the winter bees, may not represent a true seasonal shift, but may be due to the

Table IV. Levels of trypanosomatids (Crithidia mellificae/Lotmaria passim) in the sampled bees $\left(\log _{10}(\mathrm{n} / \mu \mathrm{L})\right.$ for the summer and winter samples

\begin{tabular}{llllll}
\hline Crithidia level $\log 10(\mathrm{n} / \mu \mathrm{L})$ & Not detected $<1.8$ & Low 1.8-3 & Medium 3-5 & High 5-7 & Total \\
\hline Summer foragers & 9 & 34 & 3 & 2 & 48 \\
Winter bees overall & 21 & 24 & 0 & 0 & 45 \\
Blossom honey & 6 & 9 & 0 & 0 & 15 \\
Wheat starch syrup & 6 & 9 & 0 & 0 & 15 \\
Sucrose syrup & 9 & 6 & 0 & 0 & 15 \\
\hline
\end{tabular}

The winter samples are further distinguished by treatment (feed type) 


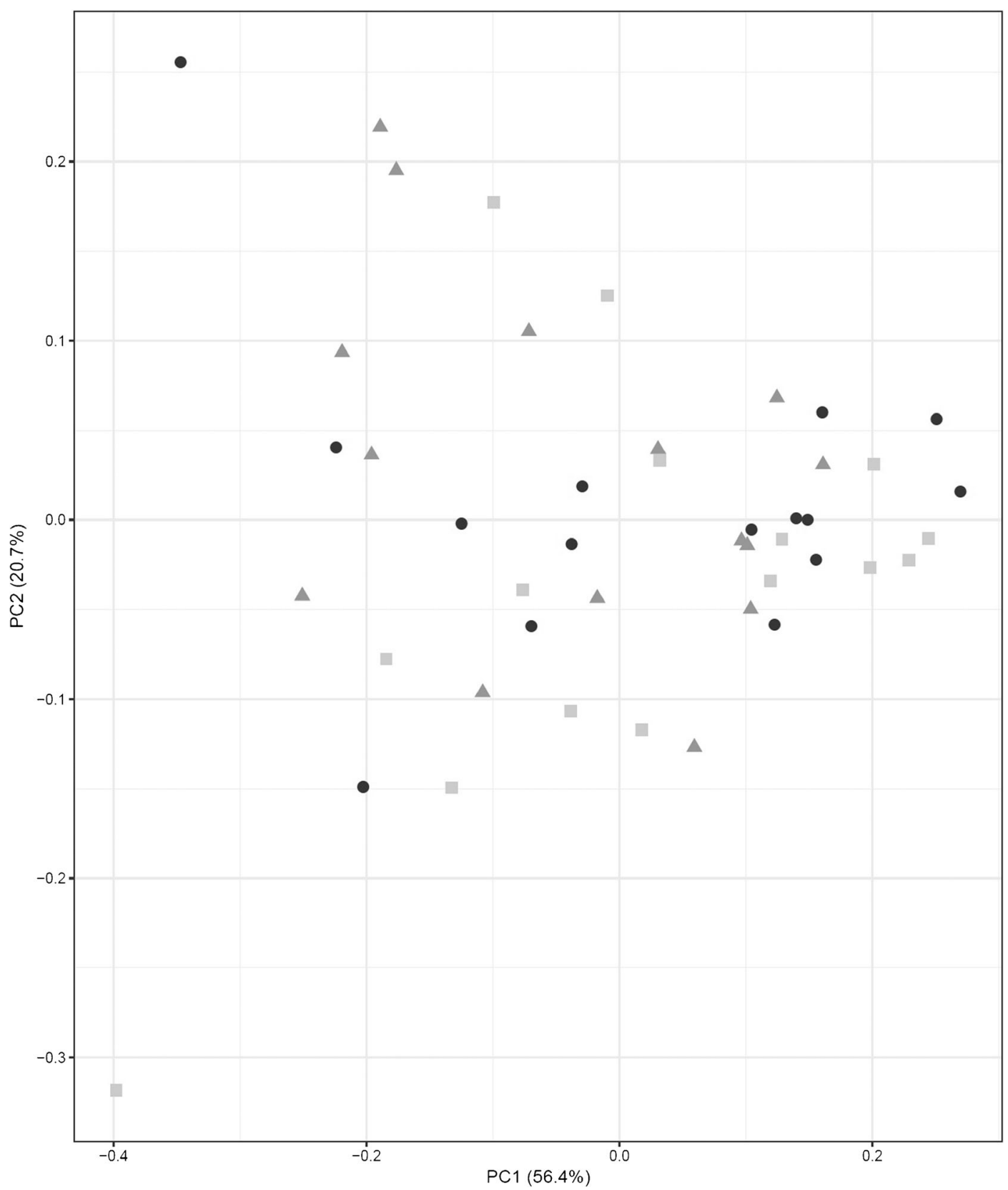

Figure 4. Principal coordinate analysis of the bacterial communities on genus level in the samples collected in winter using the Bray-Curtis method as distance metric. Circles represent samples from colonies fed with sucrose syrup, squares represent samples from colonies fed with wheat starch syrup, and triangles represent samples from colonies fed with blossom honey. A triangulation test did not indicate significant separation of clusters $(P>0.05)$.

accumulation of gut contents in the fecal bladder during winter, since Lactobacillus spp. are more abundant in the rectal lumen, while $S$. alvi has its specific niche on the ileal epithelium (Martinson et al. 2012). The surprisingly large Pseudomonas spec. fraction in some individual summer forager bees might be due to water foraging. Pseudomonas spp. are generally abundant in environmental 
freshwater sources and may, due to their opportunistic metabolic abilities and hardiness, become established in the bee gut when the intrinsic bacterial community is "watered down." Also other transient species than Pseudomonas (others) were generally more abundant in the summer foragers, which may be explained with their increased contact with the outer environment.

Our finding that $N$. ceranae was more abundant in summer than in winter is remarkable, since the infection dynamics of $N$. ceranae are often described to be seasonal, as in N. apis, with low prevalence during summer and increasing prevalence during winter (Fries et al. 2013; Retschnig et al. 2017; Traynor et al. 2016). However, in summer, we sampled foragers only, which are, as opposed to the winter bees in February, at the end of their lifespan and not required to live very long. Our previous investigations indicated a tendency towards higher pathogen levels in foragers than in younger nurse bees from the same colony (unpublished data). Other possible explanations for lower $N$. ceranae levels in the winter samples could be the higher temperature optimum and increased sensitivity to low temperature shortterm exposure of $N$. ceranae, as compared to N. apis (Gisder et al. 2010; Martín-Hernandez et al. 2009). Nevertheless, a recent study from a temperate climate area (VA, USA) found lower prevalence of $N$. ceranae in summer than in winter (Traver et al. 2012). However, since in the present study the colonies were only sampled at two time points, the seasonal aspect of the data should not be overemphasized.

In response to the different winter diets, we found only small differences in relative abundance of Rhizobiales (Bartonella apis) and Bifidobacteria. Since it is still largely unknown what roles these bacteria play for their hosts, we can only speculate what effects any changes may have. It is hard to imagine that an increase of the Bifidobacteria fraction from roughly 1 to $2 \%$ could have a notable impact on the individual bee, let alone the colony. Yet, the increase from roughly 0.5 to $15 \%$ in the Rhizobiales (Bartonella) fraction in response to feeding with honey might possibly make a difference, if the bacterium has unique and essential functions. In contrast to the Bartonella spp. that are vectored by blood-sucking insects and act as human pathogens, B. apis was never described as a pathogen and seems to assume the ancestral role of the genus as a benign insect symbiont, which is, judging from its genome, able to synthesize amino acids and cofactors (Segers et al. 2017). Whether B. apis supplies its host with these essential nutrients and whether any of its symbiotic functions are unique within the bee microbiota is not known.

It has to be pointed out that we did not find any large-scale alterations of the gut microbiota, like disappearance of a species, emergence of significant amounts of atypical bacteria, or notable proliferation of a parasite or pathogen. Therefore, the hypothesis that replacement diets cause essential changes in the intestinal microbial community has to be rejected. Yet, since our analysis cannot detect fluctuations at the strain level, there may have been fine-scale alterations of the microbiota that may, due to different metabolic abilities of the strains, have a physiological effect on the bees.

\section{CONCLUSIONS}

In summary, our results indicate that the general practice of removing honey from the hives and replacing it with a similar carbohydrate source leads to very little change in the microbiota and does not adversely affect the microbial balance or parasite levels in the intestines of the bees. This finding is in line with a previous study by Severson and Erickson (1984), who found that carbohydrate replacement diets did not affect overwintering success and colony performance in the following year. However, it remains possible that the winter feed type may have impacts on other aspects of physiology that were not yet addressed.

\section{ACKNOWLEDGEMENTS}

We thank Daniel Pfauth for his help with the bee hives.

Contributions $\mathrm{MH}, \mathrm{PR}, \mathrm{SN}, \mathrm{PD}$ conceived and designed the study; PD, AS, FB conducted experiments; SN, MB, AS, MC, PD analyzed data; PD, MH wrote the paper. All authors read and approved the final manuscript. 
Funding information This study was supported by a grant of the Ministry of Rural Development and Consumer Protection Baden-Württemberg to Peter Rosenkranz and Martin Hasselmann (MicroBee project). Sven Nahnsen and Marius Cosmin Codrea acknowledge funding from the Deutsche Forschungsgemeinschaft (core facility initiative, KO-2313/6-1 and KO-2313/6-2, institutional strategy of the University of Tuebingen, ZUK 63).

\section{COMPLIANCE WITH ETHICAL STANDARDS}

Conflict of interest The authors declare that they have no conflict of interest.

Influence du type de nourrissage hivernal sur la flore intestinale et les parasites chez les abeilles

miel / sirop / microbiome / Nosema / Crithidia

Der Einfluß des Winterfutters auf intestinale Mikrobiota und Parasiten bei Honigbienen

\section{Honig / Sirup / Mikrobiota / Nosema / Crithidia}

\section{REFERENCES}

Anderson, K. E., Sheehan, T. H., Eckholm, B. J., Mott, B. M., DeGrandi-Hoffman, G. (2011) An emerging paradigm of colony health: microbial balance of the honey bee and hive (Apis mellifera ). Insectes Soc. 58, 431-44

Baffoni, L., Gaggia, F., Alberoni, D., Cabbri, R., Nanetti, A., Biavati, B., di Gioia, D. (2016) Effect of dietary supplementation of Bifidobacterium and Lactobacillus strains in Apis mellifera L. against Nosema ceranae. Benef. Microbes 7 (1), 45-51

Cariveau, D. P., Elijah Powell, J., Koch, H., Winfree, R., Moran, N. A. (2014) Variation in gut microbial communities and its association with pathogen infection in wild bumble bees (Bombus ). ISME J. 8 (12), 1-11

Corby-Harris, V. (2016) Parasaccharibacter apium, gen. nov., sp. nov., improves honey bee (Hymenoptera: Apidae) resistance to Nosema. J. Econ. Entomol. 109 (2), 537-43

Cox-Foster, D. L., Conlan, S., Holmes, E. C., Palacios, G., Evans, J. D., et al. (2007) A metagenomic survey of microbes in honey bee colony collapse disorder. Science 318 (October), 283-87

da Silva, P. M., Gauche, C., Gonzaga, L. V., Costa, O. C. A., Fett, R. (2016) Honey: chemical composition, stability and authenticity. Food Chem. 196, 309-23

Di Prisco, G., Desiderato, A., Margiotta, M., Ferrara, R., Varricchio, P., Zanni, V. (2016) A mutualistic symbiosis between a parasitic mite and a pathogenic virus undermines honey bee immunity and health. Proc. Natl. Acad. Sci. 113 (12), 3203-8

Dillon, R., Vennard, C., Buckling, A., Charnley, A. (2005) Diversity of locust gut bacteria protects against pathogen invasion. Ecol. Lett. 8, 1291-98

Engel, P., James, R. R., Koga, R., Kwong, W. K., Quinn, S., et al. (2013) Standard methods for research on Apis mellifera gut symbionts. J. Apic. Res. 52 (4), 1-24

Engel, P., Stepanauskas, R., Moran, N. A. (2014) Hidden diversity in honey bee gut symbionts detected by single-cell genomics. PLoS Genet. 10 (9)

Engel, P., Bartlett, K. D., Moran, N. (2015a) The bacterium Frischella perrara causes scab formation in the gut of its honeybee host. MBio 6 (3), 1-8

Engel, P., Vizcaino, M. I., Crawford, J. M. (2015b) Gut symbionts from distinct hosts exhibit genotoxic activity via divergent colibactin biosynthesis pathways. Appl. Environ. Microbiol. 81 (4), 1502-12

Evans, J. D., Aronstein, K., Chen, Y. P., Hetru, C., Imler, J., et al. (2006) Immune pathways and defence mechanisms in honey bees Apis mellifera. Insect Mol. Biol. 15 (5), 645-56

Forsgren, E., Olofsson, T., Vasquez, A., Fries, I. (2010) Novel lactic acid bacteria inhibiting Paenibacillus larvae in honey bee larvae. Apidologie 41, 99-108

Fries, I., Chauzat, M., Chen, Y., Doublet, V., Genersch, E., et al. (2013) Standard methods for Nosema research. J. Apic. Res. 52 (1), 1-28

Genersch, E., Von Der Ohe, W., Kaatz, H., Schroeder, A., Otten, C., et al. (2010) The German bee monitoring project: a long term study to understand periodically high winter losses of honey bee colonies. Apidologie 41, 332-52

Gisder, S., Hedtke, K., Mo, N., Frielitz, M., Linde, A., Genersch, E. (2010) Five-year cohort study of Nosema spp. in Germany: does climate shape virulence and assertiveness of Nosema ceranae? Appl. Environ. Microbiol. 76 (9), 3032-38

Herbig, A., Maixner, F., Bos, K. I., Krause, J., Huson, D. H. (2016) MALT: fast alignment and analysis of metagenomic DNA sequence data applied to the Tyrolean Iceman. bioRxiv

Huson, D., Weber, N. (2013) Microbial community analysis using MEGAN. Methods Enzymol. 531, 465-85

Jiang, H., Lei, R., Ding, S., Zhu, S. (2014) Skewer: a fast and accurate adapter trimmer for next-generation sequencing paired-end reads. BMC Bioinformatics 15 (182), 1-12

Koch, H., Schmid-Hempel, P. (2011) Socially transmitted gut microbiota protect bumble bees against an intestinal parasite. Proc. Natl. Acad. Sci. 108 (48), 19288-92

Koch, H., Abrol, D. P., Li, J., Schmid-Hempel, P. (2013) Diversity and evolutionary patterns of bacterial gut associates of corbiculate bees. Mol. Ecol. 22, 2028-44

Kunieda, T., Fujiyuki, T., Kucharski, R., Foret, S., Ament, S. A., et al. (2006) Carbohydrate metabolism genes 
and pathways in insects: insights from the honey bee genome. Insect Mol. Biol. 15 (5), 563-76

Kwong, W. K., Moran, N. A. (2016) Gut microbial communities of social bees. Nat. Rev. Microbiol. 14 (6), 374-84

Kwong, W. K., Engel, P., Koch, H., Moran, N. A. (2014) Genomics and host specialization of honey bee and bumble bee gut symbionts. Proc. Natl. Acad. Sci. 111 (31), 11509-14

Kwong, W. K., Medina, L. A., Koch, H., Sing, K., Jia, E., Soh, Y., Ascher, J. S., Jaffé, R., Moran, N. A. (2017) Dynamic microbiome evolution in social bees. Sci. Adv. 3 (e1600513), 1-17

Maes, P. W., Rodrigues, P. A., Oliver, R., Mott, B. M., Anderson, K. E. (2016) Diet-related gut bacterial dysbiosis correlates with impaired development, increased mortality and Nosema disease in the honeybee (Apis mellifera ). Mol. Ecol. 25, 5439-50

Martín-Hernandez, R., Aranzazu, M., García-Palencia, P., Marín, P., Botías, C., Garrido-Bailo, E., Barrios, L., Higes, M. (2009) Effect of temperature on the biotic potential of honeybee Microsporidia. Appl. Environ. Microbiol. 75 (8), 2554-57

Martinson, V. G., Danforth, B. N., Minckley, R. L., Rueppell, O., Tingek, S., Moran, N. A. (2011) A simple and distinctive microbiota associated with honey bees and bumble bees. Mol. Ecol. 20, 619-28

Martinson, V., Moy, J., Moran, N. A. (2012) Establishment of characteristic gut bacteria during development of the honeybee worker. Appl. Environ. Microbiol. 78 (8), 2830-40

Meeus, I., De Graaf, D. C., Jans, K., Smagghe, G. (2010) Multiplex PCR detection of slowly-evolving trypanosomatids and neogregarines in bumblebees using broad-range primers. J. Appl. Microbiol. 109, $107-15$

Moran, N. A. (2015) Genomics of the honey bee microbiome. Curr. Opin. Insect Sci. 10, 22-28

Powell, J. E., Martinson, V. G., Urban-Mead, K., Moran, A. (2014) Routes of acquisition of the gut microbiota of the honey bee Apis mellifera. Appl. Environ. Microbiol. 80 (23), 7378-87

Retschnig, G., Williams, G. R., Schneeberger, A., Neumann, P. (2017) Cold ambient temperature promotes Nosema spp. intensity in honey bees (Apis mellifera). Insects 8 (1), 1-12

Romanelli, A., Moggio, L., Montella, C., Campiglia, P., Iannaccone, M., Capuano, F., Capparelli, R. (2011) Peptides from royal jelly: studies on the antimicrobial activity of jelleins, jelleins analogs and synergy with temporins. J. Pept. Sci. 17, 348-52

Ryu, A. J., Kim, S., Lee, H., Bai, J. Y., Nam, Y., et al. (2008) Innate immune homeostasis by the homeobox gene caudal and commensal-gut mutualism in Drosophila. Science 319 (5864), 777-82
Scarborough, C., Ferrari, J., Godfray, H. (2005) Aphid protected from pathogen by endosymbiont. Science 310, 1781

Schluter, J., Foster, K. R. (2012) The evolution of mutualism in gut microbiota via host epithelial selection. PLoS Biol. 10 (11)

Schwarz, R. S., Moran, N. A., Evans, J. D. (2016) Early gut colonizers shape parasite susceptibility and microbiota composition in honey bee workers. Proc. Natl. Acad. Sci. 113 (33), 9345-50

Segers, F. H. I. D., Ke, L., Kosoy, M., Engel, P. (2017) Genomic changes associated with the evolutionary transition of an insect gut symbiont into a bloodborne pathogen. ISME J. in press, 1-13

Severson, D. W., Erickson, E. H. (1984) Honey bee (Hymenoptera: Apidae) colony performance in relation to supplemental carbohydrates. J. Econ. Entomol. 77 (6), 1473-78

Silverman, N., Paquette, N. (2008) The right resident bugs. Science 319 (5864), 734-35

Traver, B. E., Williams, M. R., Fell, R. D. (2012) Comparison of within hive sampling and seasonal activity of Nosema ceranae in honey bee colonies. J. Invertebr. Pathol. 109 (2), 187-93

Traynor, K., Rennich, K., Forsgren, E., Rose, R., Pettis, J., et al. (2016) Multiyear survey targeting disease incidence in US honey bees. Apidologie 47, 325-47

vanEngelsdorp, D., Evans, J. D., Saegerman, C., Mullin, C., Haubruge, E., et al. (2009) Colony collapse disorder: a descriptive study. PLoS One 4 (8)

Vásquez, A., Forsgren, E., Fries, I., Paxton, R. J., Flaberg, E., Szekely, L., Olofsson, T. C. (2012) Symbionts as major modulators of insect health: lactic acid bacteria and honeybees. PLoS One 7 (3)

Viuda-Martos, C., Ruiz-Navajas, Y., Fernandez-Lopez, J., Perez-Alvarez, J. (2008) Functional properties of honey, propolis, and royal jelly. J. Food Sci. 73 (9), 11724

Wheeler, M. M., Robinson, G. E. (2014) Diet-dependent gene expression in honey bees: honey vs. sucrose or high fructose corn syrup. Sci. Rep. 4 (5726), 1-5

Wilfert, L., Long, G., Legget, H. C., Schmid-Hempel, P., Butlin, R., Martin, S., Boots, M. (2016) Deformed wing virus is a recent global epidemic in honeybees driven by Varroa mites. Science 351 (6273), 594-97

Zheng, H., Nishida, A., Kwong, W. K., Engel, P., Steele, M. I., and Moran, N. A. (2016) Metabolism of toxic sugars by strains of the bee gut symbiont Gilliamella apicola . MBio 7 (6), 1-9

Zheng, H., Powell, J. E., Steele, M. I., Dietrich, C., Moran, N. A. (2017) Honeybee gut microbiota promotes host weight gain via bacterial metabolism and hormonal signaling. Proc. Natl. Acad. Sci. 114 (18), 4775-80 